\title{
Estimation of the rate of partial inbreeding
}

\author{
Philip W. Hedrick
}

Division of Biological Sciences, University of Kansas, Lawrence, Kansas 66045, U.S.A.

Estimates are given for the rate of partial inbreeding when a maternal homozygous genotype and her progeny are known. Also considered are estimates for X-linked genes (or haplo-diploid organisms), situations in which there is more than one type of inbreeding, inbreeding in previous generations, variation in male allelic frequencies, and null alleles. These procedures could be used in animals as well as plants although the size of the progeny array must be fairly large.

\section{INTRODUCTION}

The breeding system of an organism can have a large effect on the extent and pattern of genetic variation and consequently the potential for adaptive change in the species. For example, the extent of inbreeding and outcrossing can change the heterozygosity expected within a population (e.g., Hedrick and Cockerham, 1986). Furthermore, depending upon the mode of selection, inbreeding can either increase or decrease the rate of response to a change in the environment (e.g., Hedrick, 1985).

Estimation procedures for the amount of outcrossing in predominantly self-fertilising species are well known (see Clegg, 1980 for a review). However, many species may have substantial proportions of matings between other close relatives. Matings between relatives, such as sibs or cousins, will also affect heterozygosity although to a lesser extent than self-fertilisation. Below I will discuss an extension of the direct approach of estimating the amount of self-fertilisation to the estimation of the frequency of other mating types. This method involves progeny testing of homozygous maternal genotypes obtained at random from a population polymorphic for these loci. The maximum likelihood approach is used to estimate the level of inbreeding in the population based on the numbers of each genotype observed in the progeny. Although such techniques are generally applied to plant populations, they should in many cases be applicable to estimation of inbreeding in animal populations given an adequate progeny size and a known maternal parent.

\section{METHODS OF ESTIMATION}

\section{Autosomal loci}

Assume that a proportion $S_{0}$ of the progeny are produced by self-fertilisation and $T\left(=1-S_{0}\right)$ by outcrossing, and that we are examining a locis with alleles $A_{1}$ and $A_{2}$ having frequencies $p$ and $q$, respectively. If the maternal parent is $A_{1} A_{1}$, then the proportions of progeny are as given in table 1 . Symbolising the observed number of heterozygous progeny as $N_{12}$, then

$$
N_{12}=T q N
$$

where there are $N$ total progeny and $T q N$ is the expected number of heterozygous progeny. (If the parent is $A_{2} A_{2}$, then $p$ can be substituted for $q$ and the same expressions used.) The maximum likelihood estimate of the outcrossing rate is

$$
\hat{T}=\frac{N_{12}}{q N} .
$$

(Bailey, 1951). If $q$ is known, then

$$
V(\hat{T})=\frac{\hat{T}(1-q \hat{T})}{N q} .
$$


If an independent estimate of $q$ is obtained experimentally and it is assumed that there is no covariance between $\hat{T}$ and $\hat{q}$, then

$$
V(\hat{T}) \cong \frac{\hat{T}(1-\hat{q} \hat{T})}{N \hat{q}}+\frac{V(\hat{q}) \hat{T}^{2}}{\hat{q}^{2}}
$$

(Marshall and Brown, 1974).

Table 1 The progeny and their frequencies with an $A_{1} A_{1}$ maternal parent when there is $S_{0}$ self-fertilisation and $T$ outcrossing

\begin{tabular}{lllll}
\hline & & & \multicolumn{2}{c}{ Progeny } \\
& Male parent & Frequency & $A_{1} A_{1}$ & $A_{1} A_{2}$ \\
\hline$S_{0}$ & $A_{1} A_{1}$ & 1 & $S_{0}$ & - \\
$T$ & $A_{1}$ & $p$ & $T p$ & - \\
& $A_{2}$ & $q$ & - & $T q$ \\
& & & $1-T q$ & $T q$ \\
\hline
\end{tabular}

Let us now examine a population in which a proportion $S_{1}$ of the progeny are produced by full-sib mating and $T\left(=1-S_{1}\right)$ by outcrossing. Again, assuming an $A_{1} A_{1}$ maternal parent, there are three possible genotypes for the full-sib male parent whose frequencies can be calculated in the following manner. Because the maternal parent is $A_{1} A_{1}$, then both her parents have given her an $A_{1}$ allele. Therefore, the probabilities that her sib would get an $A_{1}$ or $A_{2}$ are $\frac{1}{2}(1+p)$ and $\frac{1}{2} q$, respectively. Note that these probabilities assume that there has been no inbreeding in the immediately previous generation, the generation of the parents of the sibs. Later we will relax this assumption and show how an estimate can still be made with inbreeding in this and previous generations. Using these probabilities, the frequency of the three male full-sib genotypes are given in table 2 with the frequency of their progeny. Again, assuming that the observed number of heterozygous progeny is $N_{12}$, then

$$
N_{12}=\frac{1}{2}(1+T) q N \text {. }
$$

The estimate of outcrossing is

$$
\hat{T}=\frac{2 N_{12}}{q N}-1
$$

and if $q$ is known, then

$$
V(\hat{T})=\frac{\hat{T}(2-q \hat{T})}{N q}
$$

(after Marshall and Brown, 1974). If an independent estimate of $q$ is obtained experimentally, then

$$
V(\hat{T}) \cong \frac{\hat{T}(2-\hat{q} \hat{T})}{N \hat{q}}+\frac{V(\hat{q}) \hat{T}^{2}}{\hat{q}^{2}} .
$$

Assume that a proportion of the population $S_{2}$ is produced by half-sib mating and $T\left(=1-S_{2}\right)$ by outcrossing. With an $A_{1} A_{1}$ maternal parent, the progeny are as given in table 3 . The observed number of heterozygous progeny is

$$
N_{12}=\frac{1}{4}(3+T) q N
$$

and the estimate of outcrossing is

$$
\hat{T}=\frac{4 N_{12}}{q N}-3
$$

and if $q$ is known, then

$$
V(\hat{T})=\frac{\hat{T}(4-q \hat{T})}{N q} .
$$

If an independent estimate of $q$ is obtained, then

$$
V(\hat{T})=\frac{\hat{T}(4-\hat{q} \hat{T})}{N \hat{q}}+\frac{V(\hat{q}) \hat{T}^{2}}{\hat{q}^{2}} .
$$

Generalising from the above expressions, then if a population has a proportion $S_{i}$ of the $i$ th degree of mating where $i=0$ indicates selfing, $i=1$ full-sib mating, $i=2$ half-sib mating, etc., then

and

$$
N_{12}=\left(\frac{1}{2}\right)^{i}\left(2^{i}-1+T\right) q N
$$

$$
\hat{T}=\frac{2^{i} N_{12}}{q N}-2^{i}+1
$$

Table 2 The progeny and their frequency with an $A_{1} A_{1}$ maternal parent when there is $S_{1}$ full-sibbing and $T$ outcrossing

\begin{tabular}{lllll}
\hline & & & \multicolumn{2}{c}{ Progeny } \\
& Male parent & Frequency & $A_{1} A_{1}$ & $A_{1} A_{2}$ \\
\hline$S_{1}$ & $A_{1} A_{1}$ & $\frac{1}{4}(1+p)^{2}$ & $\frac{1}{4} S_{1}(1+p)^{2}$ & - \\
& $A_{1} A_{2}$ & $\frac{1}{2}(1+p) q$ & $\frac{1}{4} S_{1}(1+p) q$ & $\frac{1}{4} S_{1}(1+p) q$ \\
& $A_{2} A_{2}$ & $\frac{1}{4} q^{2}$ & - & $-\frac{1}{4} S_{1} q^{2}$ \\
$T$ & $A_{1}$ & $p$ & - & $T p$ \\
& $A_{2}$ & $q$ & $-1-\frac{1}{2}(1+T) q$ & $\frac{1}{2}(1+T) q$ \\
\hline
\end{tabular}


Table 3 The progeny and their frequency with an $A_{1} A_{1}$ maternal parent when there is $S_{2}$ half-sibbing and $T$ outcrossing

\begin{tabular}{lllll} 
& & & \multicolumn{2}{c}{ Progeny } \\
& Male parent & Frequency & $A_{1} A_{1}$ & $A_{1} A_{2}$ \\
\hline$S_{2}$ & $A_{1} A_{1}$ & $\frac{1}{2} p(1+p)$ & $\frac{1}{2} S_{2} p(1+p)$ & - \\
& $A_{1} A_{2}$ & $\frac{1}{2} q(1+2 p)$ & $\frac{1}{4} S_{2} q(1+2 p)$ & $\frac{1}{4} S_{2} q(1+2 p)$ \\
& $A_{2} A_{2}$ & $\frac{1}{2} q^{2}$ & - & $\frac{1}{2} S_{2} q^{2}$ \\
$T$ & $A_{1}$ & $p$ & $T p$ & - \\
& $A_{2}$ & $q$ & - & $T q$ \\
& & & & \\
& & & &
\end{tabular}

and if $q$ is known

$$
V(\hat{T})=\frac{\hat{T}\left(2^{i}-q \hat{T}\right)}{N q}
$$

If an independent estimate of $q$ is obtained, then

$$
V(\hat{T}) \cong \frac{\hat{T}\left(2^{i}-\hat{q} \hat{T}\right)}{N \hat{q}}+\frac{V(\hat{q}) \hat{T}^{2}}{\hat{q}^{2}}
$$

\section{X-linked loci or haplo-diploid organisms}

Let us also examine the estimate of inbreeding for $\mathrm{X}$-linked loci or haplo-diploid organisms. For fullsib mating, the frequency of $A_{1}$ and $A_{2}$ males are $\frac{1}{2}(1+p)$ and $\frac{1}{2} q$, respectively, giving the progeny frequencies in table 4 so that

$$
N_{12}=\frac{1}{2}(1+T) q N
$$

and

$$
\hat{T}=\frac{2 N_{12}}{q N}-1
$$

which is the same as for autosomal genes. For partial half-sib mating there are two types of matings. Let $S_{2}^{\prime}$ and $S_{2}^{\prime \prime}$ be the proportions for the situations in which there is a common female parent and a common male parent, respectively. The frequencies of males and progeny are given in table 5 so that

$$
N_{12}=\left(1-\frac{1}{2} S_{2}^{\prime}\right) q N
$$

If it is assumed that $S_{2}^{\prime}=S_{2}^{\prime \prime}=\frac{1}{2} S_{2}$, then

$$
\hat{T}=\frac{4 N_{12}}{q N}-3
$$

making this result also identical to the autosomal estimate.

\section{More than one type of inbreeding}

Assume that more than one type of inbreeding occurs in the population, then the number of heterozygotes from an $A_{1} A_{1}$ maternal parent is

$$
N_{12}=\left(1-\sum_{i=0}^{n}\left(\frac{1}{2}\right)^{i} S_{i}\right) q N \text {. }
$$

If we assume a given heterozygosity level in the progeny $\left(H=N_{12} / N\right)$, then this heterozygosity can be the result of different proportions of partial inbreeding.

The equilibrium inbreeding coefficient when there are different types of partial inbreeding is

$$
f_{e}=\frac{S_{i}}{2^{i+1}-\left(2^{i+1}-1\right) S_{i}}
$$

Hedrick and Cockerham (1986). However, different combinations of $S_{i}$ values that give the same proportion of heterozygous progeny can result in different equilibrium inbreeding coefficients in expression (12). For example, in the first row of table $6, H=0.25$ can be the result of $\hat{S}_{0}=0.5$ or $\hat{S}_{1}=1 \cdot 0$. Using expression (12) these

Table 4 The progeny and their frequencies with an $A_{1} A_{1}$ maternal parent for an X-linked locus (or a haplo-diploid organism) when there is $S_{1}$ full-sib mating and $T$ outcrossing

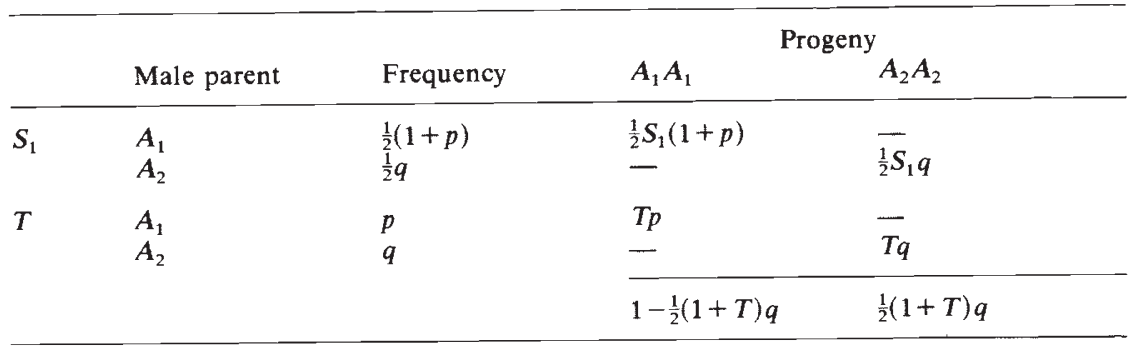


Table 5 The progeny and their frequencies with an $A_{1} A_{1}$ maternal parent for an X-linked locus (or haplo-diploid) when there is $S_{2}^{\prime}$ and $S_{2}^{\prime \prime}$ of the two types of half-sib mating and $T$ outcrossing

\begin{tabular}{lllll}
\hline & & & \multicolumn{2}{c}{ Progeny } \\
& Male parent & Frequency & $A_{1} A_{1}$ & $A_{1} A_{2}$ \\
\hline$S_{2}^{\prime}$ & $A_{1}$ & $\frac{1}{2}(1+p)$ & $\frac{1}{2} S_{2}^{\prime}(1+p)$ & - \\
& $A_{2}$ & $\frac{1}{2} q$ & - & $\frac{1}{2} S_{2}^{\prime} q$ \\
$S_{2}^{\prime \prime}$ & $A_{1}$ & $p$ & $S_{2}^{\prime \prime} p$ & - \\
& $A_{2}$ & $q$ & - & $S_{2}^{\prime \prime} q$ \\
$T$ & $A_{1}$ & $p$ & $T p$ & - \\
& $A_{2}$ & $q$ & - & $T q$ \\
\hline
\end{tabular}

levels result in $f_{\mathrm{e}}$ values of $0 \cdot 333$ and $1 \cdot 0$, respectively.

\section{Other factors affecting the estimates}

Past inbreeding When we derived the estimates above, we assumed that there was no inbreeding in the parental generation of the sibs, half-sibs, etc. In fact, there may well have been inbreeding making the frequency of $A_{1} A_{1}$ higher than what we assumed and the frequency of $A_{1} A_{2}$ lower. To illustrate how this affects the estimates, let us assume in the partial full-sib estimation that the frequencies of genotypes in the parental generation (parents of the maternal genotype) are $p^{2}+f p q$, $2 p q(1-f)$, and $q^{2}+f p q$ for $A_{1} A_{1}, A_{1} A_{2}$, and $A_{2} A_{2}$, respectively. Because the maternal genotype is $A_{1} A_{1}$, then the frequencies of these two genotypes in her parents are expected to be $p+f q$ and $q-f q$ for $A_{1} A_{1}$ and $A_{1} A_{2}$, respectively. Notice that if $f=0$ we get the frequencies we used before and if $f=1$, then all parents are $A_{1} A_{1}$.

The expected frequencies of the full-sib male are then given in table 7 as a function of the inbreeding coefficient in the parents of the full sibs. The number of $A_{1} A_{2}$ progeny is then

$$
N_{12}=\frac{1}{2} q(1+T-f-f T) N
$$

so that

$$
\hat{T}=\frac{2 N_{12}-q N(1-f)}{q N(1+f)} .
$$

If $f=0$, then this expression reduces to (3) and if $f=1$, then

$$
\hat{T}=\frac{N_{12}}{q N}
$$

which is equal to (1), the estimate for self-fertilisation. In other words, if $f=1$, then all full-sib matings produce $A_{1} A_{1}$ homozygous progeny. This actually improves the estimate of outcrossing because there are no $A_{1} A_{2}$ progeny produced by full-sib mating.

If we assume that partial inbreeding has occurred for several generations, then the inbreeding coefficient should be near the inbreeding equilibrium value given by (12) (Hedrick, 1986). For example, if we assume that there has been $S_{2}$ full-sib mating then $f_{e}=S_{2} /\left(4-3 S_{2}\right)$. If we let $q=$ $0.5, S_{2}=0.25(T=0.75)$ so that $f_{e}=0.077$, then from (13), $H=0.404$. This value compares with $H=0.4375$ (see table 6) if the population had not been inbred in the past. In other words, the same level of partial inbreeding over several generations will result in fewer heterozygotes observed than if

Table 6 The level of partial inbreeding that can account for a given heterozygosity in the progeny from a $A_{1} A_{1}$ maternal parent and the resulting inbreeding equilibrium when $q=0.5$

\begin{tabular}{llll}
\hline & & $H$ & \\
& $0 \cdot 25$ & $0 \cdot 375$ & $0 \cdot 4375$ \\
\hline$\hat{S}_{0}, f_{e}$ & $0 \cdot 5,0 \cdot 333$ & $0 \cdot 25,0 \cdot 142$ & $0 \cdot 125,0 \cdot 067$ \\
$\hat{S}_{1}, f_{e}$ & $1 \cdot 0,1 \cdot 0$ & $0 \cdot 5,0 \cdot 2$ & $0 \cdot 25,0 \cdot 077$ \\
$\hat{S}_{2,}, f_{e}$ & - & $1 \cdot 0,1 \cdot 0$ & $0 \cdot 5,0 \cdot 111$ \\
$\hat{S}_{0}=\hat{S}_{1}, f_{e}$ & $0 \cdot 333,0 \cdot 429$ & $0 \cdot 167,0 \cdot 158$ & $0 \cdot 083,0 \cdot 070$ \\
$\hat{S}_{0}=\hat{S}_{2}, f_{e}$ & $0 \cdot 4,0 \cdot 556$ & $0 \cdot 2,0 \cdot 172$ & $0 \cdot 1,0 \cdot 072$ \\
$\hat{S}_{1}=\hat{S}_{2}, f_{e}$ & - & $0 \cdot 333,0 \cdot 273$ & $0 \cdot 167,0 \cdot 086$ \\
\hline
\end{tabular}


Table 7 The progeny and their frequency with an $A_{1} A_{1}$ maternal parent when there is $S_{1}$ full-sibbing,

$T$ outcrossing, and an inbreeding coefficient of $f$ in the parents of the maternal parent

\begin{tabular}{lllll} 
& Male parent & Frequency & $A_{1} A_{1}$ & $A_{1} A_{2}$ \\
\hline$S_{1}$ & $A_{1} A_{1}$ & $\frac{1}{4}(1+p+f q)^{2}$ & $\frac{1}{4} S_{1}(1+p+f q)^{2}$ & - \\
& $A_{1} A_{2}$ & $\frac{1}{2} q(1+p+f q)(1-f)$ & $\frac{1}{4} q S_{1}(1+p+f q)(1-f)$ & $\frac{1}{4} q S_{1}(1+p+f q)(1-f)$ \\
& $A_{2} A_{2}$ & $\frac{1}{4} q^{2}(1-f)$ & - & $\frac{1}{4} q^{2} S_{1}(1-f)^{2}$ \\
$T$ & $A_{1}$ & $p$ & $T p$ & $-\overline{T q}$ \\
& $A_{2}$ & $q$ & - & $\frac{1}{2} q(1+T-f+f T)$ \\
\hline
\end{tabular}

the inbreeding only occurred in the generation being examined. A potential problem arises if the level of past inbreeding is not known and all the heterozygosity is assumed due to inbreeding in the examined generation. In the example above, this would lead to an estimate that $S_{2}=0.384$, over 50 per cent higher than the actual value. One way to avoid such a problem would be to carry out a population survey of genotypic frequencies to determine if there was a deficiency of heterozygotes in the population. Of course, the effect above is due to inbreeding in a previous generation so contemporary information would only be suggestive of inbreeding in the earlier generation.

Variation in male allelic frequency Assume that the allelic frequency among half the males is $q-\Delta q$ and among the other half is $q-\Delta q$. If there is only selfing, then the number of heterozygous progeny is

$$
\begin{aligned}
N_{12} & =\left[\frac{1}{2} T(q+\Delta q)+\frac{1}{2} T(q-\Delta q)\right] N \\
& =T q
\end{aligned}
$$

In other words, variation in pollen frequencies makes no difference in the mean estimation of outcrossing for partial selfing.

However, for partial full-sib and higher degrees of inbreeding, variation in male allelic frequencies does affect the outcrossing estimates. For partial full-sib mating

$$
\begin{aligned}
N_{12}= & {\left[\frac{1}{8} S_{1}(1+p-\Delta q)(q+\Delta q)+\frac{1}{8} S_{1}(1+p+\Delta q)\right.} \\
& \times(q-\Delta q)+\frac{1}{8} S_{1}(q+\Delta q)^{2}+\frac{1}{8} S_{1}(q-\Delta q)^{2} \\
& \left.+\frac{1}{2} T(q+\Delta q)+\frac{1}{2} T(q-\Delta q)\right] N \\
= & {\left[\frac{1}{2}(1+T) q+\frac{1}{8} S_{1}(\Delta q)^{2}\right] N . }
\end{aligned}
$$

In this case, the maximum effect is when $\Delta q=0.5$ implying that $q=0.5$. If we let $S_{1}=0.5$ then $N_{12}$ is approximately four per cent larger than if there were no variation in $q$. This results in an increase in the estimate of outcrossing of 12.8 per cent $(0.564$ versus 0.5$)$. Similar calculations can be carried out for other inbreeding levels, suggesting that variation in allelic frequency has a small effect on outcrossing estimates. The variance of these estimates should be larger than if there was a constant $q$.

Null alleles Assume that a null allele for $A_{n}$ has a frequency $r$ so that any outcrossed $A_{1} A_{n}$ progeny would be scored as $A_{1} A_{1}$ progeny. For partial selfing, the difference between the observed and actual outcrossing rates is

$$
\begin{aligned}
\Delta T & =\frac{N_{12}}{N q}-\frac{N_{12}}{N(q+r)} \\
& =-\frac{r N_{12}}{q(q+r) N} .
\end{aligned}
$$

For partial full-sib mating,

$$
\begin{aligned}
\Delta T & =\frac{2 N_{12}}{q N}-1-\left[\frac{2 N_{12}}{(q+r) N}-1\right] \\
& =-\frac{2 r N_{12}}{q(q+r) N} .
\end{aligned}
$$

In other words, the estimates of outcrossing are reduced by $r /[q(q+r)]$ : If $q=0.5$ and $r=0.01$, then this leads to an underestimate of outcrossing of 3.9 per cent. Brown (1979) discusses how null alleles can influence the fixation index for partialselfing populations, and suggests that nulls should not constitute a problem with proper precaution.

\section{DISCUSSION}

The estimates given here illustrate a technique to estimate the extent of inbreeding other than selffertilisation. To detect low levels of inbreeding, large numbers of individuals are necessary. For example, for low values of partial full-sib mating, say $S_{1}=0.05$, then at least 3000 progeny must be scored to statistically show $S_{1}>0$ (after Marshall and Brown, 1974). It appears that when $S_{1}>0 \cdot 2$, the sample size in progeny tests for some organisms becomes reasonable $(N<100)$. 
As discussed in Hedrick and Cockerham (1986), the deficiency of heterozygotes seen in outbreeders by Brown (1979) may be the result of consanguineous matings. This can be understood using the values in table 6 . For example, assume that $H=0.375$ which could be the result of partial selfing of 0.25 that results in an equilibrium inbreeding $f_{e}$ of $0 \cdot 142$. However, if the level of inbreeding was really the result of full sib and half sib mating so that $S_{1}=S_{2}=0.333$ (last line of table 6 ), then $f_{e}=0 \cdot 273$. In this case, the observed level of heterozygosity in the population would be higher than that expected by the selfing estimate $(0 \cdot 273-0 \cdot 142=0 \cdot 131)$.

Recently, several studies have examined the estimation of partial inbreeding other than selfing. For example, Ritland (1984) has derived estimates of "effective selfing" which includes as a group different degrees of relatedness among mates. Schoen and Clegg (1984) compared the situations where the outcrossed progeny were full sibs instead of half sibs. Hopefully, by either expanding the mixed-mating model or by closely evaluating its assumptions as in these studies, we will develop a better understanding of mating systems in natural populations (e.g., Brown et al., 1985).

\section{REFERENCES}

BAILEY, N. T. S. 1951. Testing the solubility of maximum likelihood equations in the routine application of scoring methods. Biometrics, 7, 268-274.

BROWN, A. H. D. 1979. Enzyme polymorphism in plant populations. Theoret. Pop. Biol., 15, 1-42.

BROWN, A. H. D., BARRETT, S. C. H. AND MORAN, G. F. 1985. Mating system estimation in forest trees: Models, methods, and meanings. Gregorius, H. -R. (ed.) In Population Genetics in Forestry, Lecture Notes in Biomathematics, 60 , Springer-Verlag, New York, pp. 32-49.

ClEGG, M. 1980. Measuring plant mating systems. Bioscience, 30, 814-818.

HEDRICK, P. W. 1985. Inbreeding and selection in natural populations, Gregorius, H. -R. (ed.) In Population Genetics in Forestry, Lecture Notes in Biomathematics 60, SpringerVerlag, New York, pp. 71-91.

HEDRICK, P. W. 1986. Average inbreeding or inbreeding equilibrium? Amer. J. Hum. Genet., 38, 965-970.

HEDRICK, P. W. AND COCKERHAM, C. C. 1986. Partial inbreeding: Equilibrium heterozygosity and the heterozygosity paradox. Evolution, 40, 856-861.

MARSHALL, D. R. AND BROWN, A. H. D. 1974. Estimation of the level of apomixis in plant populations. Heredity, 32, 321-333.

RITLAND, K. 1984. The effective proportion of self-fertilisation with consanguineous matings in inbred populations. Genetics, 106, 139-152.

SCHOEN, D. J. AND CLEGG, M. T. 1984. Estimation of mating system parameters when outcrossing events are correlated. Proc. Natl. Acad. Sci., 81, 5258-5262. 